\title{
Recombinant myxoma virus infection associated with high mortality in rabbit farming (Oryctolagus cuniculus)
}

\author{
Fábio A. Abade dos Santos ${ }^{1}$, Carina Carvalho ${ }^{2}$, Madalena Monteiro ${ }^{2}$, Paulo Carvalho ${ }^{2}$, \\ Paula Mendonça ${ }^{2}$, M. Conceição Peleteiro ${ }^{1}$, and Margarida Duarte ${ }^{2}$ \\ ${ }^{1}$ University of Lisbon Faculty of Veterinary Medicine \\ ${ }^{2}$ INIAV
}

September 9, 2020

\begin{abstract}
Myxomatosis is an emergent disease in Iberian hare, having been considered a rabbit disease for decades. Genome sequencing of the strains obtained from affected Iberian hares showed to be distinct from the classical strains that circulated in rabbits since the virus introduction in Europe, in 1952. The main genomic difference concerns the presence of an additional $2.8 \mathrm{~Kb}$ region disrupting the M009L gene and adding a set of genes with homology to the MYXV genes M060R, M061, M064 and M065R originated in poxviruses. After the emergence of this recombinant virus (MYXV-Tol or ha-MYXV), in the summer of 2019, the recombinant MYXV was not detected in rabbit surveys suggesting apparent species segregation with the MYXV classic strains persistently circulating in rabbits. Recently, a group of six unvaccinated European rabbits (Oryctolagus cuniculus cuniculus) from a backyard rabbitry in the South Portugal, developed signs of myxomatosis (anorexia, dyspnoea, oedema of eyelids, head, ears, external genitals and anus, and skin myxomas in the base of the ears), five of them dying within 24-48 hours of symptoms onset. Molecular analysis revealed that only the recombinant myxoma virus was present. This is the first documented report of a recombinant myxoma virus (ha-MYXV) in farm rabbits associated with high mortality, which aggravates the concern for the future of the Iberian hare and wild rabbits and the safety of the rabbit industry against which the existing vaccines may not be fully protective.
\end{abstract}

Recombinant myxoma virus infection associated with high mortality in rabbit farming (Oryctolagus cuniculus)

Short title: Recombinant MYXV infection in European rabbit

Authors and affiliations

Fábio A. Abade dos Santos ${ }^{1,2,3}{ }^{*}$, Carina L. Carvalho ${ }^{1}$, Madalena Monteiro ${ }^{1}$, Paulo Carvalho ${ }^{1}$, Paula Mendonça $^{1}$, M. Conceição Peleteiro ${ }^{1}$, Margarida D. Duarte ${ }^{1,2}$

${ }^{1}$ INIAV, IP. National Institute of Agrarian and Veterinarian Research. Av. da República, 2780-143 Oeiras, Portugal

${ }^{2}$ CIISA, Centre for Interdisciplinary Research in Animal Health, Faculty of Veterinary Medicine of Lisbon, Avenida da Universidade Técnica 1300-477 Lisboa. Portugal

${ }^{3}$ Instituto Universitario de Biotecnología de Asturias (IUBA), Departamento de Bioquímica y Biología Molecular, Universidad de Oviedo, Oviedo, Spain

Corresponding author

Fábio A. Abade dos Santos (fabio.abade@iniav.pt) 


\section{Summary}

Myxomatosis is an emergent disease in Iberian hare, having been considered a rabbit disease for decades. Genome sequencing of the strains obtained from affected Iberian hares showed to be distinct from the classical strains that circulated in rabbits since the virus introduction in Europe, in 1952. The main genomic difference concerns the presence of an additional $2.8 \mathrm{~Kb}$ region disrupting the M009L gene and adding a set of genes with homology to the MYXV genes M060R, M061, M064 and M065R originated in poxviruses. After the emergence of this recombinant virus (MYXV-Tol, ha-MYXV), in the summer of 2019, recombinnat MYXV was not detected in rabbit surveys suggesting apparent species segregation with the MYXV classic strains persistently circulating in rabbits.

Recently, a group of six unvaccinated European rabbits (Oryctolagus cuniculus cuniculus) from a backyard rabbitry in the South Portugal, developed signs of myxomatosis (anorexia, dyspnoea, oedema of eyelids, head, ears, external genitals and anus, and skin myxomas in the base of the ears), five of them dying within 24-48 hours of symptoms onset. Molecular analysis revealed that only the recombinant myxoma virus was present.

This is the first documented report of a recombinant myxomatosis virus in farm rabbits associated with high mortality, which aggravates the concern for the future of the Iberian hare and wild rabbits and the safety of the rabbit industry against which the existing vaccines may not be fully protective.

Keywords: European rabbit, Oryctolagus cuniculus cuniculus , rabbit farming, Recombinant myxoma virus, ha-MYXV, MYXV-Tol, Myxomatosis, Myxoma virus

\section{Introduction}

Myxoma virus (MYXV) is a Leporipoxvirus from the Poxviridae family that causes an infectious, often fatal, systemic disease in the European rabbit called myxomatosis. The virus is a dsDNA that replicates in cytoplasm (Murphy et al., 1995). After the virus introducing in France, in 1952, MYXV spread throughout all Europe, with mortalities rates as high as 90\% (Fenner and Ratcliffe, 1965). Over the years, the virulence of the strains decreased significantly due to increase of genetic resistance by the host, development of immunity and natural selection of less virulent strains (Fenner and Fantini, 1999; Kerr, 2012). Nowadays, the disease persists and is considered one of the main reasons behind the fragile conservation status (danger of extinction) of the European rabbit (Duarte et al., 2018; Villafuerte and Delibes-Mateos, 2019; C. L. Carvalho et al., 2020).

The development and production of heterologous and homologous vaccines against myxomatosis for rabbits, proved to confer seroconversion in almost 100\% of rabbits (Hipra, 2003), and, along with the application of biosafety measures, allow to control the disease in the industrial rabbit farming and small rabbitries, contrarily to wild life.

During almost seven decades, myxomatosis has been rarely reported in European brown hares (Collins, 1955; Wibbelt and Frolich, 2005), never in Iberian hare, being considered a rabbit disease. However, in late 2018, a natural recombinant MYXV (ha-MYXV) emerged in Iberian hare, leading to the first epidemic outbreaks in this species, throughout Portugal and Spain (Bocanegra et al., 2019; C. L. Carvalho et al., 2020). The change in host tropism was attributed to a genomic modification comprising a $2.8 \mathrm{~kb}$ insertion containing genes with homology to the M060R, M061R, M064 and M065R genes of MYXV, which are located around position 60Kb in the genome. The insert disrupted the M009L gene (Dalton et al., 2019; Pinto et al., 2019) located around position $12 \mathrm{~Kb}$ in the MYXV genome, which became divided in two smaller ORFs.

To date, no cases of mortality in European rabbit due to this recombinant virus have been documented, although cases of myxomatosis in wild and domestic rabbits continue to be monitored and genotyped in Portugal, under the scope of a national surveillance program (+Coelho Project, dispatch 4575, 31 may 2017, MAFDR).

Here we report a case of high mortality ([?]83\%) in a small backyard rabbitry. Genotyping confirmed the 
unique presence of a recombinant virus, similar to the ha-MYXV identified in Iberian hares. None of the rabbits was vaccinated impeding any inference on the degree of protection conferred by commercial vaccines against the recombinant MYXV. However, the confirmation that recombinat MYXV induces severe disease in rabbits, raises new concerns for the wild and domestic leporid species, emphasizing the need of continuous monitoring and genomic characterization of viruses circulating in these species in the Iberia.

\section{Material and Methods}

\subsection{Case presentation}

In July 2020, a small rabbit farm (Aljustrel, South Portugal) of one adult male (two years old), two adult males (one year old), one adult female (one year old) and two juveniles (5 months old) European rabbits, developed signs compatible with myxomatosis. The six rabbits presented clinical signs of disease on the same day.

The adult male (two years old) showed slight signs of illness, with very slight swelling of the eyelid and slight serous discharge, recovering from disease in around one week. After the disappearing of the clinical signals, a blood sample was collected from this rabbit by jugular venepuncture (Abade dos Santos et al., 2019) to evaluate the serological response to MYXV infection using the CIVTEST Cuni Myxomatosis Kit (Hipra) according with manufacturer indications. The other five animals died 24-48 hours after the symptoms onset, which included oedema of the eyelids (Fig.1), base of the ears and genitals, dyspnoea and anorexia. Small myxomas at the base of the ear were present in one of the rabbits (Fig. 2).

\subsection{Necropsy and Histopathology}

After death, three (22388PT20, 22390PT20 and 22391PT20) of the five rabbits were necropsied according to routine procedures. Tissue samples were collected for histopathology (eyelid, genitalia, ear, lung and kidney) and virology (liver, spleen, lung, eyelid and genitalia). The other two rabbits were autolysed, therefore only submitted to virological examination.

For histopathology, liver, spleen, kidney, eyelid, ear, muzzle and lung fragments were fixated in 10\% (w/v) neutral buffered formalin, routinely paraffin embedded, sectioned at $4 \mu \mathrm{m}$, and stained with Haematoxylin and $\operatorname{Eosin}(\mathrm{H} \& \mathrm{E})$.

\subsection{Molecular analysis}

For nucleic acid extraction, fresh samples of eyelid were homogenized at $20 \%(\mathrm{w} / \mathrm{v})$ with phosphate buffered saline and clarified at 3,000g for $5 \mathrm{~min}$. Total nucleic acid were extracted from $200 \mu \mathrm{l}$ of the clarified supernatants, using the MagAttract 96 cador Pathogen Kit in a BioSprint extractor (Qiagen, Hilden, Germany), according to the manufacturer's protocol.

In the Scope of Project + Coelho, all the rabbits were tested for RHDV, RHDV2 and MYXV due to possible co-infections (Duarte et al., 2018; C. L. Carvalho et al., 2020). The rabbits were tested for rabbit haemorrhagic disease virus 2 (RHDV2) by the qPCR (Duarte et al., 2015), and for RHDV by conventional PCR (Tham et al., 1999), using the AgPath (Thermo Fisher Scientific) and One step RT-PCR kit (Qiagen, Hilden, Germany), respectively. MYXV was initial tested by the qPCR method described by (Duarte et al., 2014), which detects both MYXV and ha-MYXV virus, using the FastStart TaqMan Probe Master Kit (Roche, Germany).

The preliminary analysis for recombinat MYXV DNA was performed with the conventional PCR system 9E/9F described by Dalton et al. (Dalton et al., 2019), using the HighFidelity PCR Master Mix (Roche, Germany), according with manufacturer protocol.

Amplifications were carried out in a Bio-Rad CFX96 Thermal Cycler (Bio-Rad Laboratories Srl, Redmond, USA).

The PCR products were visualized in $2 \%$ horizontal electrophoresis agarose gels, purified with the NZYGelpure kit (Nzytech, Portugal), and directly sequenced using the ABI Prism BigDye Terminator 
v3.1 Cycle sequencing kit on a 3130 Genetic Analyser (Applied Biosystems, Foster City, CA, U.S.A). Sequencing of the $859 \mathrm{nt}$ fragment within the $2.8 \mathrm{~Kb}$ insert that includes the partial M064L and M061L gene, was performed with primers designed in this work, 1467F (5'- TTCATCGTTTATGGGAAAATCTATG-3'), 1819R (5'- GAGGGGACAGTTATGGATGTAC- 3'), 2028F (5'-AAGATGCGTCTGTGTAACAATCC-3') and 2325R (5'- AACAATGTATACACTCATGACAGTAC-3'). The nucleotide sequences obtained were assembled using the Seqscape Software v2.7 (Applied Biosystems, Foster City, CA, USA), and submitted to GenBank.

\section{Results}

\subsection{Necropsy and Histopathology}

The cadavers of all three necropsied rabbits were in good body condition suggesting a very rapid course of the disease. Necropsy confirmed the presence of $1 \mathrm{~cm}$ nodules in the base of the ears in one male rabbit (Fig. 1), together with nose, lips and external genitalia oedema, purulent blepharitis and lung densification foci. Another adult male showed white ocular discharge (Fig. 2) and focal lung congestion. Along with oedema of left eyelid, one juvenile also presented thickening foci of ears.

Histopathology of the skin lesions revealed marked epidermal hyperplasia, frequent ballooning degeneration of keratinocytes and myxoid change of the dermal connective tissue (Fig. 3). In the lung, thickening of interalveolar septa was observed due to infiltration of heterophils and mononucleated inflammatory cells, confirming interstitial pneumonia (Fig.4).

\subsection{Virological results}

All animals tested negative to RHDV and RHDV2. The male rabbit, with two years old, was seropositive with high antibody titter $(\mathrm{RI} 10=18.4)$. No serum was available from the five dead rabbits due to blood coagulation. The owner reported no previous vaccination, but being the oldest animal in the group, we cannot exclude that it may have contacted with classic MYXV strains in the past, acquiring some level of cross-immunity, allowing its survival.

The Cq values obtained with the qPCR-MYXV targeting the diploid gene M0005L/R in the tissues from three rabbits revealed high viral charges in a pool of liver and spleen (mean of 2,01E+09 copies/mg), lungs (mean of $2.25 \mathrm{E}+09$ copies $/ \mathrm{mg}$ ), eyelid (mean of $5.45 \mathrm{E}+10$ copies $/ \mathrm{mg}$ ) and genitalia (mean of $1.97 \mathrm{E}+09$ copies/mg).

The amplicon size obtained with the 9E/9F system ([?] 3100), confirmed that rabbits were infected with the recombinant MYXV and not with classic MYXV strains. Further nucleotide sequencing of an 859nt fragment from two rabbits (MT920563 and MT920564) revealed 100\% of similarity between the two sequences. BLAST analysis showed also 100\% of identity with the homologous region encompassing M061L and M064L of sequence MK340973 (ha-MYXV), confirming that rabbits were infected by the MYXV recombinant myxoma virus.

\section{Discussion}

The presence of external signs of myxomatosis in three adult and three juvenile farm European rabbits in an outbreak registered in Aljustrel (Alentejo, South Portugal) in August 2020, the clinical outcome (83\% mortality), along with the histopathological and molecular data, confirmed that five out of six infected rabbits died from this disease and that the virus involved was the recombinant myxoma virus, thus demonstrating the susceptibility of rabbits to this new virus.

Lesions described in the three necropsied rabbits, in particular skin lesions, were fully coincident with the ones of classical myxomatosis also found in hares(Carvalho et al., 2020), such as ballooning degeneration of keratinocytes and myxoid change of the dermal connective tissue.

Given that ha-MYXV, originally considered hare specific, is also being detected in rabbits, we rather refer to ha-MYXV (also known as MYXV-Tol) as recombinant myxoma virus (rec-MYXV), since it affects both 
leporid species.

The mortality found in this small backyard rabbitry was [?]83\%, apparently higher than what was estimated in Iberian hare' outbreaks (55.4\%) (García-Bocanegra et al., 2020), despite the small number of animals involved may biased this evaluation.

Interestingly, the oldest of the rabbits (a two years old male) developed very mild symptoms and survived the infection developing high antibody titter $(\mathrm{RI} 10=18.4)$. No serum was available from the five dead rabbits due to blood coagulation. The owner reported no previous vaccination, but being the oldest animal in the group, we cannot exclude that it may have contacted with classic MYXV strains in the past, acquiring some level of cross-immunity, allowing its survival.

Despite the biosecurity measures adopted in the rabbit industry, virus introduction from the outside is always at risk. The rabbit industry is one of the largest expanding meat industries in the world, having had a positive evolution of $85 \%$ between 1998 and 2017. In fact, in 2017, around 1,483,000 tons of rabbit meat were produced in the world (Trocino et al., 2019), being the China, Democratic People's Republic of Korea and Spain the bigger producers. In China, the research funding for rabbit science reaches values of 2,443 million of Euros and in France and Germany, the research in this area is funded with around 600-700 millions of Euros a year (Trocino et al., 2019).

When myxomatosis emerged in the Europe around 1950's, and until the development of vaccines, millions of domestic rabbits died with incalculable economic losses. Just in Great Britain, tens of millions of rabbits died (Bartrip, 2008). Spill over between the wild and domestic animals has been appointed as source of infection for outbreaks in the industry (C. L. Carvalho et al., 2017). Given the impact that viral infectious diseases may have in this sector, it is critical to monitor the sanitary status of industry and of the wild populations.

To the best of our knowledge this is the first documented description of myxomatosis in domestic rabbits by recombinant MYXV (referred as ha-MYXV or MYXV-Tol) infection. It is crucial to verify if commercial vaccines fully/effectively protect rabbits from this recombinant virus.

\section{Acknowledgements}

This work was financed by the Fundo Florestal Permanente, Government of Portugal, (Project + Coelho 2, ref. 2019014300001, as part of the Action Plan for the Control of Rabbit Viral Haemorrhagic Disease (Dispatch no. 4757/2017 of 31 May)). Fundação para a Ciência e Tecnologia (FCT) funded Fábio A. Abade dos Santos (SFRH/BD/137067/2018). The Centre for Interdisciplinary Research in Animal Health (CIISA) from the Faculty of Veterinary Medicine (University of Lisbon) also contributed to this study (CIISAUIDP/CVT/00276/2020).

\section{Ethics statement}

The authors confirm that the ethical policies of the journal, as noted on the journal's author guidelines page, have been adhered to. No ethical approval was required as no animal was killed for the purpose of this study.

\section{Conflict of interest}

The authors declare no competing interests.

\section{Data Sharing}

The data that support the findings of this study are available from the corresponding author upon reasonable request.

\section{References}

Abade dos Santos, F.A., C.L. Carvalho, M.C. Peleteiro, S. Gabriel, R. Patrício, F.A. Abade, C.L. Carvalho, M.C. Peleteiro, S.I. Gabriel, R. Patrício, J. Carvalho, M. V Cunha, and M.D. Duarte, 2019: Blood collection from the external jugular vein of Oryctolagus cuniculus algirus sedated with midazolam : live sampling of a 
subspecies at risk Blood collection from the external jugular vein of Oryctolagus cuniculus algirus sedated with midazolam : live. Wildlife Biol.1, DOI: 10.2981/wlb.00588.

Bartrip, P.W.J., 2008: Myxomatosis in 1950s Britain. 19 , 83-105, DOI: 10.1093/tcbh/hwm016.

Bocanegra, I.G., L. Camacho-Sillero, M.A. Risalde, K. Dalton, J. Caballero-Gomez, M. Aguero, I. Zorrila, and F. Gomez-Guillamon, 2019: First outbreak of myxomatosis in Iberian hares ( Lepus granatensis ).Transbound. Emerg. Dis. 1-5, DOI: 10.1111/tbed.13289.

Carvalho, C., F.A. Abade Dos Santos, M. Monteiro, P. Carvalho, P. Mendonca, and M.D. Duarte, 2020: First cases of myxomatosis in Iberian hares (Lepus granatensis) in Portugal. Vet. Rec. Case Reports 8 , DOI: 10.1136/vetreccr-2019-001044.

Carvalho, C.L., F.A. Abade dos Santos, T. Fagulha, P. Carvalho, P. Mendonca, M. Monteiro, and M.D. Duarte, 2020: Myxoma virus and rabbit haemorrhagic disease virus 2 coinfection in a European wild rabbit ( Oryctolagus cuniculus algirus ), Portugal. 18-21, DOI: 10.1136/vetreccr-2019-001002.

Carvalho, C.L., E. Leclerc Duarte, J.M. Monteiro, C. Afonso, J. Pacheco, P. Carvalho, P. Mendonca, A. Botelho, T. Albuquerque, P. Themudo, M. Fevereiro, A.M. Henriques, S. Santos Barros, and M. Dias Duarte, 2017: Progression of rabbit haemorrhagic disease virus 2 upon vaccination in an industrial rabbitry: A laboratorial approach. World Rabbit Sci. 25 , 73-85, DOI: 10.4995/wrs.2017.5708.

Collins, J.J., 1955: Myxomatosis in the common hare. Ir. Vet. J.9, 268 .

Dalton, K.P., J.M. Martin, I. Nicieza, A. Podadera, D. Llano, R. Casais, S. Gimenez, I. Badiola, M. Aguero, M. Duran, D. Buitrago, L.J. Romero, E. Garcia, and F. Parra, 2019: Myxoma virus jumps species to the Iberian hare. Transbound. Emerg. Dis. tbed.13296, DOI: 10.1111/tbed.13296.

Duarte, M.D., S.C. Barros, A.M. Henriques, M.T. Fagulha, F. Ramos, T. Luis, and M. Fevereiro, 2014: Development and validation of a real time PCR for the detection of myxoma virus based on the diploid gene M000 . 5L / R. J. Virol. Methods 196 , 219-224, DOI: 10.1016/j.jviromet.2013.11.014.

Duarte, M.D., C.L. Carvalho, F.A. Abade dos Santos, J. Gomes, P.C. Alves, P.J. Esteves, J. Abrantes, A.M. Lopes, M. P., A. Serronha, N. Santos, P.T. Santos, Y. Vaz, J. Carvalho, F.C. Pinto, J. Amaro, and M. V. Cunha, 2018: +Coelho: Avaliacao Ecossanitaria das Populacoes Naturais de Coelho Bravo Visando o Controlo da Doenca Hemorragica Viral. .

Duarte, M.D., C.L. Carvalho, S.C. Barros, A.M. Henriques, F. Ramos, T. Fagulha, T. Lu??s, E.L. Duarte, and M. Fevereiro, 2015: A real time Taqman RT-PCR for the detection of rabbit hemorrhagic disease virus 2 (RHDV2). J. Virol. Methods 219 , 90-95, DOI: 10.1016/j.jviromet.2015.03.017.

Fenner, F., and B. Fantini, 1999: History of Myxomatosis - an Experiment in Evolution.CABI., In: Biological Control of Vertebrate Pests. New York, USA.

Fenner, F., and F.N. Ratcliffe, 1965: Myxomatosis. Cambridge Univ. Press. London, UK.

Garcia-Bocanegra, I., L. Camacho-Sillero, J. Caballero-Gomez, M. Aguero, F. Gomez-Guillamon, J. RuizCasas, J. Diaz-Cao, E. Garcia, M. Ruano, and R. Haza, 2020: Monitoring of emerging myxoma virus epidemics in Iberian hares (. Transbound. Emerg. Dis. DOI: 10.1111/tbed.13781.

Hipra, L., 2003: SEROLOGICAL RESPONSE TO MYXOMATOSIS VACCINATION BY DIFFERENT INOCULATION SYSTEMS ON FARM RABBITS. 145-156.

Kerr, P.J., 2012: Myxomatosis in Australia and Europe: A model for emerging infectious diseases. Antiviral Res. 93 , 387-415, DOI: 10.1016/j.antiviral.2012.01.009.

Murphy, F.A., C.M. Fauquet, D.H.L. Bishop, S.A. Ghabrial, A.W. Jarvis, G.P. Martelli, M.A. Mayo, and M.. Summers, 1995: Virus taxonomy: classification and nomenclature of Viruses. . Springer Verlag, Vienna, 
Austria: Sixth report of the International Committee for the Taxonomy of Viruses. Archives of Virology, Suppl. 10.

Pinto, A., A.L. De Matos, M. Abrantes, S. Kraberger, M.A. Risalde, C. Gort, G. Mcfadden, A. Varsani, and P.J. Esteves, 2019: Genetic Characterization of a Recombinant Myxoma Virus in the Iberian Hare ( Lepus granatensis ). Viruses 11, 1-16, DOI: 10.3390/v11060530.

Tham, K.M., S.M. Barnes, and S.N. Hunter, 1999: Polymerase chain reaction amplification and gene sequence analysis of a calicivirus from a feral rabbit. Virus Genes 18 , 235-42.

Trocino, A., E. Cotozzolo, C. Zomeno, M. Petracci, G. Xiccato, C. Castellini, A. Trocino, E. Cotozzolo, C. Zomeno, and M. Petracci, 2019: Rabbit production and science : the world and Italian scenarios from 1998 to 2018. Ital. J. Anim. Sci. 18, 1361-1371, DOI: 10.1080/1828051X.2019.1662739.

Villafuerte, R., and M. Delibes-Mateos, 2019: Oryctolagus cuniculus (errata version published in 2020). IUCN Red List Threat. Species 2019 e.T41291A17061965\%. DOI: https://dx.doi.org/10.2305/IUCN.UK.2019-3.RLTS.T41291A170619657.en.

Wibbelt, G., and K. Frolich, 2005: Infectious Diseases in European Brown Hare (lepus europaeus). Wildl. Biol. Pract. 1 , DOI: 10.2461/wbp.2005.1.11.

\section{Figure captions}

Figure 1. Purulent blepharoconjunctivitis and muzzle oedema.

Figure 2. Skin nodules in the ear basis (myxomas).

Figure 3. (Left) nodular thickening corresponding to the accumulation of myxoid tissue in the dermis auricular cartilage (arrow head, digitalized slide, no magnification, HEEE). (Right) Detail of the previous figure in which the myxoide tissue $(M)$ and the star cells (arrow heads) are visible; epidermis hyperplasia and follicular epithelium (E) (100x, HËE).

Figure 4. Thickening of interalveolar septa by infiltration of predominantly mononucleated cells (arrow heads); pulmonary alveoli (A) (200x, HEE).

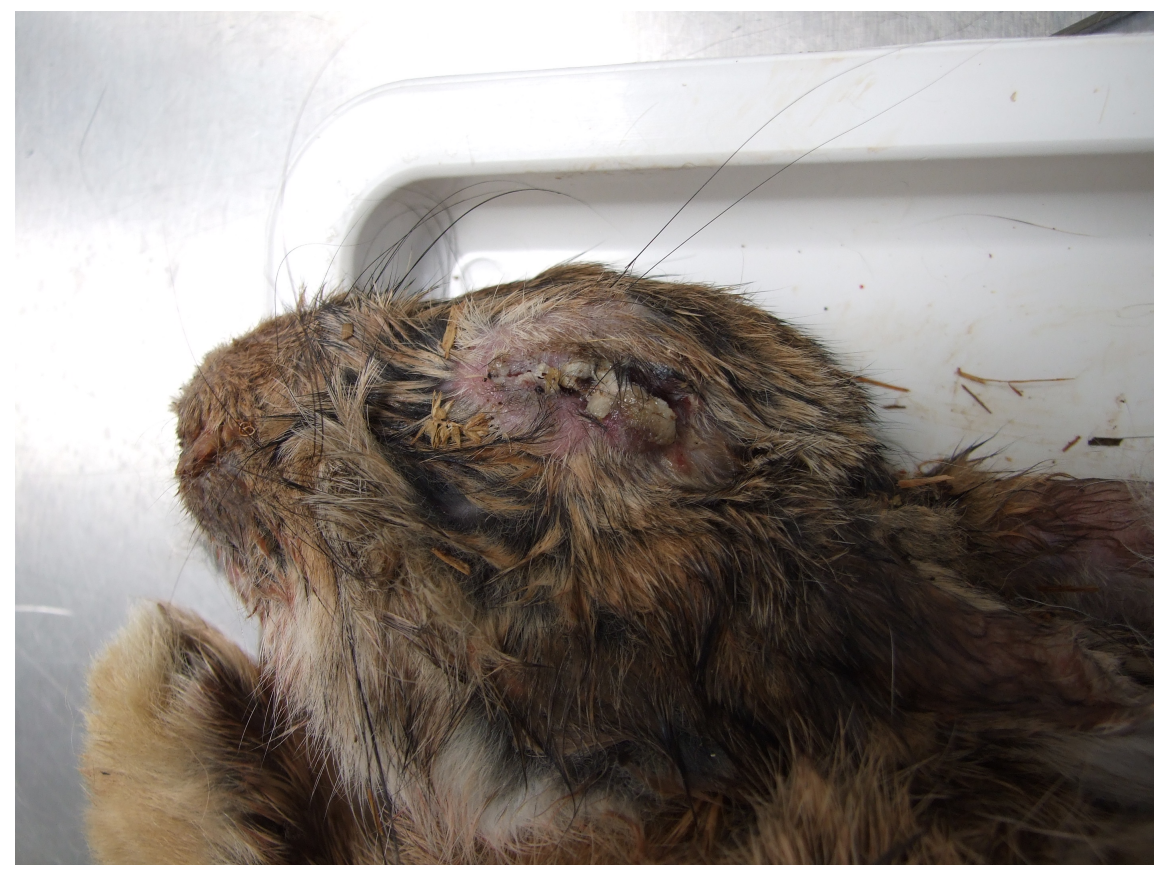



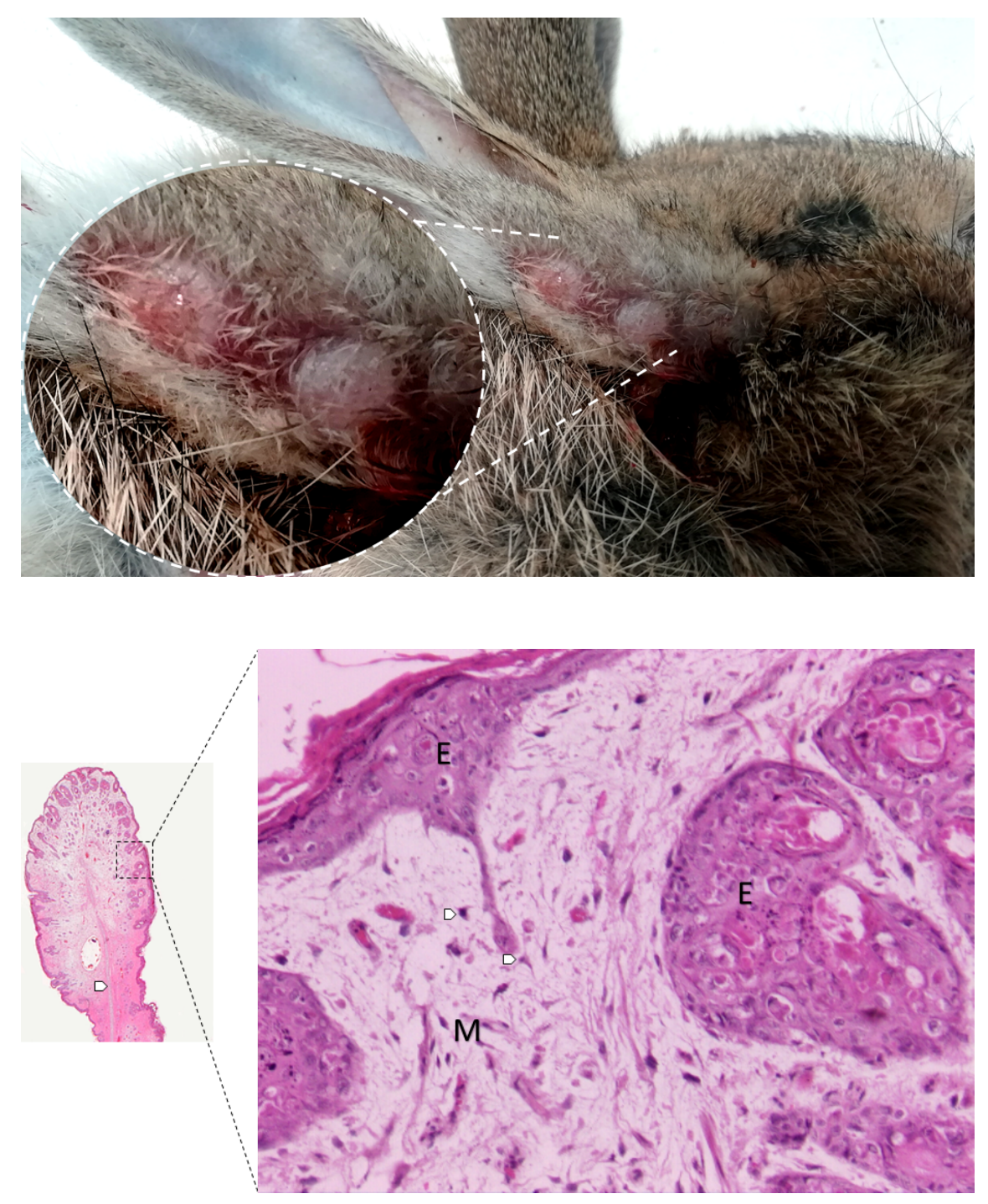


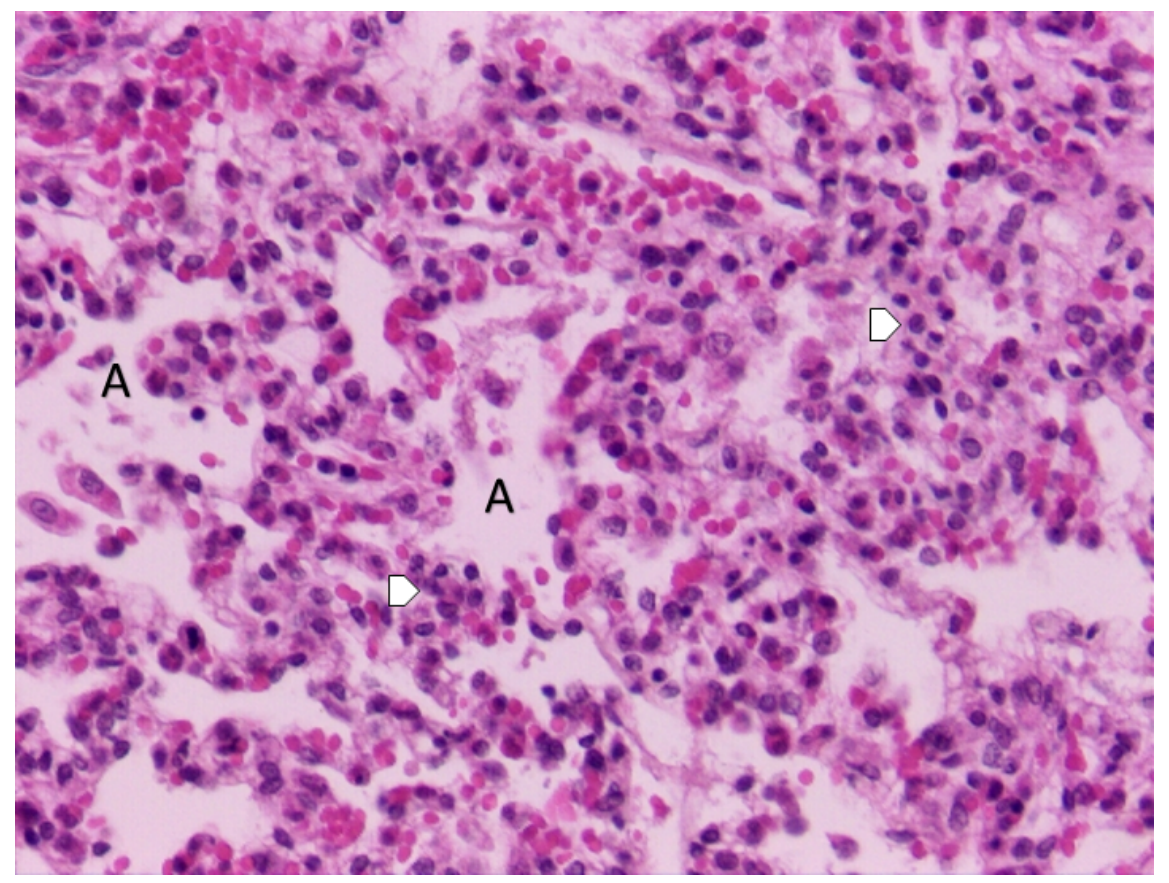

